\title{
Common versus psychopathology-specific risk factors for psychotic experiences and depression during adolescence
}

\author{
D. Kounali ${ }^{1}$ t, S. Zammit ${ }^{1,2 *}$, N. Wiles ${ }^{1}$, S. Sullivan ${ }^{1}$, M. Cannon ${ }^{3}$, J. Stochl ${ }^{4}$, P. Jones ${ }^{4}$, L. Mahedy ${ }^{2}$, \\ S. H. Gage ${ }^{1}$, J. Heron ${ }^{1}$ and G. Lewis ${ }^{1}$ \\ ${ }^{1}$ School of Social and Community Medicine, University of Bristol, UK \\ ${ }^{2}$ MRC Centre for Neuropsychiatric Genetics and Genomics, Cardiff University, Wales, UK \\ ${ }^{3}$ Royal College of Surgeons in Ireland and Beaumont Hospital, Dublin, Ireland \\ ${ }^{4}$ Department of Psychiatry, University of Cambridge, UK
}

Background. An argument often used to support the view that psychotic experiences (PEs) in general population samples are a valid phenotype for studying the aetiology of schizophrenia is that risk factors for schizophrenia show similar patterns of association with PEs. However, PEs often co-occur with depression, and no study has explicitly tested whether risk factors for schizophrenia are shared between PEs and depression, or are psychopathology specific, while jointly modelling both outcomes.

Method. We used data from 7030 subjects from a birth cohort study. Depression and PEs at age 18 years were assessed using self-report questionnaires and semi-structured interviews. We compared the extent to which risk factors for schizophrenia across sociodemographic, familial, neurodevelopmental, stress-adversity, emotional-behavioural and substance use domains showed different associations with PEs and depression within bivariate models that allowed for their correlation.

Results. Most of the exposures examined were associated, to a similar degree, with an increased risk of both outcomes. However, whereas female sex and family history of depression showed some discrimination as potential risk factors for depression and PEs, with stronger associations in the former, markers of abnormal neurodevelopment showed stronger associations with PEs.

Conclusions. The argument that PEs are valid markers for studying the aetiology of schizophrenia, made simply on the basis that they share risk factors in common, is not well supported. PEs seem to be a weak index of genetic and environmental risk for schizophrenia; however, studies disentangling aetiological pathways to PEs from those impacting upon co-morbid psychopathology might provide important insights into the aetiology of psychotic disorders.

Received 23 September 2013; Revised 20 December 2013; Accepted 20 December 2013; First published online 31 January 2014

Key words: ALSPAC, depression, epidemiology, psychotic experiences, risk factors.

\section{Introduction}

Psychotic experiences (PEs) such as delusions and hallucinations can be elicited using semi-structured interviews in approximately $5 \%$ of adolescents and young adults in the general population (Linscott \& van Os, 2013). Delusions and hallucinations are key diagnostic features of schizophrenia, and studying PEs may increase our understanding of how schizophrenia develops if PEs in general population

\footnotetext{
* Address for correspondence: Dr S. Zammit, Department of Psychological Medicine and Neurology, MRC Centre for Neuropsychiatric Genetics and Genomics, School of Medicine, Cardiff University, Heath Park, Cardiff CF14 4XN, Wales, UK (Email: zammits@cardiff.ac.uk)

† These authors served as joint first authors.
}

samples result from aetiological mechanisms underlying schizophrenia.

PEs during childhood are associated with an increased risk of schizophrenia spectrum and other psychotic disorders during adult life (Hanssen et al. 2005; Welham et al. 2009b; Werbeloff et al. 2012; Fisher et al. 2013; Zammit et al. 2013b), but the positive predictive value of such experiences is low (Poulton et al. 2000; Dhossche et al. 2002; Dominguez et al. 2011; Werbeloff et al. 2012; Zammit et al. 2013b). Consequently, evidence often used to support the view that PEs constitute a valid phenotype for studying the aetiology of schizophrenia is that risk factors for schizophrenia (Malmberg et al. 1998; Mortensen et al. 1999; Cannon et al. 2002b; McGrath et al. 2004; Wicks et al. 2005; Moore et al. 2007; Morgan \& Fisher, 2007; van Os \& Kapur, 2009; Welham et al. 2009a) 
show similar patterns of association with PEs, with reports that PEs are associated with male sex (van Os et al. 2009; Kelleher et al. 2012a), family history of schizophrenia (Krabbendam et al. 2004; Polanczyk et al. 2010), migrant or ethnic minority status (van Os et al. 2009), urbanicity (Dominguez et al. 2010; Polanczyk et al. 2010), socio-economic disadvantage (van Os et al. 2009; Polanczyk et al. 2010; Zammit et al. 2013b), pregnancy and birth complications (Zammit et al. 2009), developmental impairments (Cannon et al. 2002a), lower IQ (Cannon et al. 2002a; Horwood et al. 2008), stressful and traumatic experiences (Arseneault et al. 2011), peer victimization (Schreier et al. 2009), poorer social adjustment (Polanczyk et al. 2010) and cannabis use (Moore et al. 2007).

However, the overlap in the epidemiology of PEs with that for schizophrenia is not entirely consistent, with some studies reporting that PEs are observed more frequently in women (van Os et al. 2000; Breetvelt et al. 2010; Binbay et al. 2012; Nuevo et al. 2012; Zammit et al. 2013b) or in those living in rural areas (Wiles et al. 2006; Bartels-Velthuis et al. 2010), or failing to find evidence of increased risk of PEs in those with a family history of schizophrenia (Zammit et al. 2008). Even where epidemiological findings for schizophrenia and PEs are consistent, it is questionable to what extent these demonstrate evidence of shared aetiology, given that most of the factors associated with increased risk of schizophrenia and PEs are also associated with other psychiatric outcomes. For example, socio-economic adversity (Lorant et al. 2003), pregnancy and birth complications (Nosarti et al. 2012), developmental delay and cognitive impairment (van Os et al. 1997), autistic traits (Lundstrom et al. 2011), emotional problems and interpersonal difficulties during childhood (Cannon et al. 2002a), stressful or traumatic events and victimization (Thapar et al. 2012) and cannabis use (Moore et al. 2007) are also associated with increased risk of depression.

In fact, co-morbidity with depression is one of the most consistent findings regarding PEs in general population samples (Dhossche et al. 2002; Scott et al. 2009; Armando et al. 2010; van Rossum et al. 2011; Varghese et al. 2011; Kelleher et al. 2012a). One interpretation of this is that PEs might be a manifestation of depression rather than an indication of abnormal pathology underlying schizophrenia-related disorders. Understanding how best to conceptualize PEs is important because this has implications for classification of PEs and for predicting their utility in aiding our understanding of mechanisms underlying the aetiology of schizophrenia.

Studies examining latent constructs of psychopathology show that depression and psychosis exist as separate, but strongly correlated, dimensions in the general population (Stefanis et al. 2002; Krabbendam et al. 2004). Given the degree of co-morbidity, it is therefore not unexpected that most risk factors for PEs are also associated with risk of depression, as described earlier. Although some studies have examined risk factors for both depression and PEs within the same study (Krabbendam et al. 2004; Breetvelt et al. 2010), as far as we are aware no study has explicitly tested whether risk factors are shared between PEs and depression or are psychopathology specific while jointly modelling both outcomes to account for their co-morbidity. Examining to what extent risk factors for schizophrenia show similar, or different, patterns of association with PEs compared to depression can help to shed light on how useful studies of PEs might be in helping us understand the aetiology of schizophrenia.

In this study we compared the extent to which risk factors for schizophrenia across sociodemographic, familial, neurodevelopmental, stress and adversity, emotional-behavioural and substance use domains show different associations with PEs compared to with depression. We used statistical methods that account for the correlation between PEs and depression to avoid the problem of similar associations for PEs and depression resulting from co-morbidity of these two outcomes.

\section{Method \\ Participants}

The sample comprised participants from the Avon Longitudinal Study of Parents and Children (ALSPAC; www.alspac.bris.ac.uk), an ongoing population-based study investigating a wide range of influences on health and development of children (Boyd et al. 2012). Ethical approval for the study was obtained from the ALSPAC Law and Ethics Committee and the Local Research Ethics Committees. From the initial core sample of 14541 pregnancies, 13988 children were alive at 12 months. Among these, 7030 children had at least one measure of interest (depression or PE measures at either childhood or early adulthood) and were included in the analysis.

\section{Measures}

Depression outcome at 18 years

The depression outcome measure at 18 years used in this study was based on the computerized revised Clinical Interview Schedule (CIS-R; Lewis et al. 1992; Lewis, 1994), a fully structured interview designed for community samples. The computerized version shows very close agreement with the interviewer-administered 
version (Patton et al. 1999; Bell et al. 2005). The assessment asks questions about the week prior to interview and the onset and duration of each episode. The depression symptom score reflects the severity of depression symptoms and was calculated as the sum of the scores for the following CIS-R items: depression, depressive thoughts, fatigue, concentration and sleep (score range: 0-21). As depression can be considered as a continuum, we used a cut-off of 10 to indicate the presence of depression. Selection of this cut-off resulted in a prevalence of cases that was the same as the prevalence of PEs, thus aiding comparison of probit scores and odds ratios (ORs) for these two outcomes.

PEs at 18 years

PEs at 18 years were assessed using the PsychosisLike Symptom interview (PLIKSi; Horwood et al. 2008; Zammit et al. 2013b). The PLIKSi is a semistructured instrument that draws on the principles of standardized clinical examination developed for the Schedule for Clinical Assessment in Psychiatry (SCAN) and covers hallucinations (visual and auditory), delusions (spied on, persecution, thoughts read, reference, control, grandiosity) and experiences of thought interference (broadcasting, insertion and withdrawal). Any unspecified delusions elicited were also rated. Cross-questioning was used to establish the presence of symptoms, and coding followed glossary definitions and rating rules for the SCAN. Interviewers rated experiences as not present, suspected or definitely psychotic that had occurred since age 12 years. Unclear responses after probing were always 'rated down', and symptoms only rated as definite when a clear example was provided. PEs were coded as present in this study if one or more of the experiences was either 'suspected or definitely present' and if they could not be directly attributed to falling asleep/waking or to fever.

\section{Exposures}

Based on evidence from previous literature that these were associated with schizophrenia, we examined 19 risk factors across the following six domains (see Table 1 and Supplementary online Appendix 1 for more detail):

(1) Sociodemographic: gender, ethnicity, maternal education, maternal marital status, maternal home ownership, urbanicity.

(2) Familial: family history of depression and family history of schizophrenia.

(3) Neurodevelopmental: hypoxia at birth, gross motor development (and change) assessed at 6 and 18 months, cognitive function at 8 years, and autistic spectrum traits characterized between 6 months and 9 years.

(4) Stress-adversity: life events at 9 years and victimization at 8 years.

(5) Emotional-behavioural: Strength and Difficulties Questionnaire (SDQ) domains for conduct, hyperactivity-inattention and peer relationship problems; the Development and Well Being Assessment (DAWBA) for anxiety at age 10; the Short Mood and Feelings Questionnaire (SMFQ) for depressive symptoms at 12 years; the PLIKSi for PEs at 12 years.

(6) Substance use: cannabis use up to the age of 16 years.

\section{Statistical analysis}

Bivariate probit regression models were used to jointly model the binary indicators of depression and $\mathrm{PE}$ at 18 years and to test for equality of regression parameters expressing the effect of exposures on each outcome using likelihood ratio tests. For this purpose we compared a model that allowed effect estimates to differ for our outcomes with a model where the exposure effect was constrained to be the same for both outcomes. We converted probit estimates into ORs to enable presentation of results in a format that would be more easily interpretable. This was achieved by obtaining approximations of the logit parameters by multiplying the probit parameters by a factor of 1.6 (see Amemiya 1981, eq. 2.7; Ntzoufras et al. 2003). As a sensitivity analysis, we also examined the effect of exposures (omitting cannabis use and previous measures of PEs and depression) on a multivariate outcome that used data on these outcomes across both ages 12 and 18 .

There were significant amounts of missing data on both outcomes. Missing depression or PE outcome data at 18 years was strongly associated with gender, socio-economic position and early loss of follow-up. To deal with missing data, we used multiple imputation (MI; Little \& Rubin, 2002) by fully conditional specification using flexible additive imputation models as implemented in the aregImpute function in the $\mathrm{R}$ statistical package (Harell, 2001; van Buuren et al. 2006). In addition to those included in the analyses, the imputation model also included variables that were associated with missingness or were predictive of depression or PE at 18 years. Approximately 60 auxiliary variables were included in the imputation model to make the basic assumption underlying MI realistic. These included a wide range of markers of sociodemographic characteristics during early childhood and historical assessments of most exposures examined (e.g. SDQ scores were assessed repeatedly 
Table 1. Description of exposures under study

\begin{tabular}{|c|c|c|c|c|}
\hline Measure & Scale & Analysis units & Sample $n$ & $n(\%)$ exposed \\
\hline \multicolumn{5}{|l|}{ Sociodemographic } \\
\hline Ethinicity & Binary & Non-white & 6641 & $277(4.2)$ \\
\hline Urbanicity & Binary & Rural & 6958 & $449(6.5)$ \\
\hline Sex & Binary & Female & 7030 & $3626(51.6)$ \\
\hline Marital status of mother & Binary & Not married & 6866 & $1292(18.8)$ \\
\hline Low maternal education & Binary & CSEs & 6504 & $662(9.42)$ \\
\hline Home ownership & Binary & Renting & 6800 & $1164(17.12)$ \\
\hline \multicolumn{5}{|l|}{ Familial } \\
\hline Family history of depression & Binary & Present & 6749 & $1922(28.5)$ \\
\hline Family history of schizophrenia & Binary & Present & 5773 & $166(2.88)$ \\
\hline \multicolumn{5}{|l|}{ Neurodevelopmental } \\
\hline Hypoxia & Binary & Present & 6449 & $558(8.7)$ \\
\hline Gross motor score at 6 months & $z$ score $^{\mathrm{a}}$ & S.D. scale & 6067 & \\
\hline Gross motor score at 18 months & $z$ score $^{\mathrm{a}}$ & S.D. scale & 6297 & \\
\hline IQ at 8 years & WISC (45-151) & $\operatorname{NED}(1 \text { s.D. }=15)^{\mathrm{b}}$ & 5811 & \\
\hline Autistic spectrum disorder & Binary & $<-1$ & 6957 & $1104(15.7)$ \\
\hline \multicolumn{5}{|l|}{ Stress and adversity } \\
\hline \multirow[t]{3}{*}{ Life events at 7 years } & $0,1, \geqslant 2$ & 0 & 5882 & $4210(71.6)$ \\
\hline & & 1 & & $1376(23.4)$ \\
\hline & & $\geqslant 2$ events & & $296(5.0)$ \\
\hline Victimization at 8 years & Binary & Present & 5517 & $2164(39.2)$ \\
\hline \multicolumn{5}{|l|}{ Emotional-behavioural } \\
\hline Conduct score at 12 years & $0-10$ & 1 s.D. $=2^{\mathrm{a}}$ & 5662 & \\
\hline Hyperactivity score at 12 years & $0-10$ & 1 s.D. $=2^{\mathrm{a}}$ & 5651 & \\
\hline Peer problems score at 12 years & $0-10$ & 1 s.D. $=2^{\mathrm{a}}$ & 5663 & \\
\hline Depression at 12 years & $0-26$ & $>11$ score & 6344 & $579(9.13)$ \\
\hline Anxiety disorder at 10 years & Binary & Present & 5774 & $110(1.91)$ \\
\hline PEs at 12 years & Binary & Present & 6448 & $745(11.6)$ \\
\hline \multicolumn{5}{|l|}{ Substance use } \\
\hline \multirow[t]{4}{*}{ Cannabis use at 16 years } & Lifetime count & Never & 4102 & $2920(71.2)$ \\
\hline & & $<20$ times & & $854(20.8)$ \\
\hline & & $20-60$ & & $159(3.9)$ \\
\hline & & $>60$ & & $169(4.12)$ \\
\hline
\end{tabular}

CSE, Certificate of Secondary Education; WISC, Wechsler Intelligence Scale for Children; NED, normal equivalent deviates; PE, psychotic experience.

${ }^{a}$ Analysis scale is expressed in standard deviation (S.D.) units where the scale is transformed to have a mean 0 and S.D. $=1$.

${ }^{\mathrm{b}}$ NED - standardized scale. Raw scores are transformed to $z$ scores (standard normal scores) using the inverse normal function.

at ages 47 and 81 months, and 8, 9, 10, 11 and 13 years). Further information is available from the authors. Parameter estimates were averaged over 100 imputed datasets using Rubin rules. Wald-type tests were used following imputation to assess equality or commonality of exposure effects.

\section{Results}

\section{Descriptive}

Of the 7030 individuals in the analysis sample, 4140 had data on PEs at age 18 years. Of these, 370
[9.0\%, 95\% confidence interval (CI) 8.2-9.9] had at least one PE present (see Zammit et al. 2013b for further details). Of 4435 individuals who had data on depression at age 18 years, 410 (9.2\%, 95\% CI 8.4-10.1) had a depression score of $>10$ (cut-off selected to ensure similar prevalence of both outcomes). Among those with PEs at age 18 years, 28\% also had depression; and among those with depression, $28 \%$ also had PEs.

The correlation between depression and PE was 0.20 (95\% CI $0.09-0.31)$ at age 12 and 0.22 (95\% CI 0.09-0.36) at age 18, and this co-occurrence was 
Table 2. Exposure effects (OR and 95\% CI) on depression and psychotic experiences (PEs) at 18 years ${ }^{\mathrm{a}}$, and examination of whether psychopathology-specific effects differ from a common effect

\begin{tabular}{|c|c|c|c|c|c|c|c|c|}
\hline & \multirow[b]{2}{*}{$n$} & \multicolumn{2}{|c|}{ Depression } & \multicolumn{2}{|l|}{ PEs } & \multicolumn{3}{|c|}{ Common effect } \\
\hline & & OR & $95 \% \mathrm{CI}$ & OR & $95 \% \mathrm{CI}$ & OR & $95 \% \mathrm{CI}$ & $p$ value $^{\mathrm{b}}$ \\
\hline \multicolumn{9}{|l|}{ Sociodemographic } \\
\hline Ethnicity (non-White) & 3916 & 1.42 & $0.95-2.12$ & 1.44 & $0.97-2.16$ & 1.43 & $1.05-1.96$ & 0.942 \\
\hline Urbanicity (rural) & 4060 & 0.90 & $0.64-1.28$ & 0.73 & $0.50-1.05$ & 0.81 & $0.62-1.07$ & 0.347 \\
\hline Sex (female) & 4100 & 1.99 & $1.64-2.38$ & 1.34 & $1.12-1.60$ & 1.61 & $1.41-1.86$ & $<0.001$ \\
\hline Maternal status (unmarried) & 4035 & 1.58 & $1.28-1.95$ & 1.74 & $1.41-2.14$ & 1.66 & $1.41-1.95$ & 0.467 \\
\hline Maternal education (low) & 3847 & 1.27 & $0.94-1.71$ & 1.57 & $1.18-2.09$ & 1.42 & $1.13-1.78$ & 0.253 \\
\hline Home ownership (rented) & 3988 & 1.60 & $1.28-1.99$ & 1.67 & $1.34-2.08$ & 1.63 & $1.37-1.94$ & 0.745 \\
\hline \multicolumn{9}{|l|}{ Familial } \\
\hline Family history of depression & 3970 & 1.45 & $1.21-1.75$ & 1.10 & $0.91-1.34$ & 1.27 & $1.10-1.47$ & 0.023 \\
\hline Family history of schizophrenia & 3571 & 1.57 & $0.98-2.53$ & 1.33 & $0.81-2.18$ & 1.45 & $0.99-2.12$ & 0.584 \\
\hline \multicolumn{9}{|l|}{ Neurodevelopmental } \\
\hline Asphyxia & 3766 & 0.79 & $0.56-1.11$ & 1.30 & $0.96-1.75$ & 1.03 & $0.81-1.32$ & 0.015 \\
\hline Gross motor levels & 3402 & 1.04 & $0.98-1.11$ & 1.08 & $1.02-1.15$ & 1.06 & $1.01-1.11$ & 0.323 \\
\hline Gross motor differentials & 3402 & 0.98 & $0.90-1.06$ & 1.12 & $1.03-1.22$ & 1.05 & $0.98-1.11$ & 0.011 \\
\hline IQ & 3498 & 1.00 & $0.91-1.10$ & 0.88 & $0.79-0.97$ & 0.94 & $0.87-1.01$ & 0.037 \\
\hline Autistic spectrum disorder & 4100 & 0.89 & $0.70-1.14$ & 1.29 & $1.03-1.61$ & 1.08 & $0.90-1.03$ & 0.016 \\
\hline \multicolumn{9}{|l|}{ Stress and adversity } \\
\hline Life events & 3525 & 1.14 & $0.99-1.32$ & 1.13 & $0.98-1.32$ & 1.14 & $1.01-1.27$ & 0.952 \\
\hline Victimization & 3339 & 1.42 & $1.17-1.72$ & 1.50 & $1.23-1.83$ & 1.46 & $1.25-1.70$ & 0.659 \\
\hline \multicolumn{9}{|l|}{ Emotional-behavioural } \\
\hline Conduct & 3389 & 1.24 & $1.13-1.37$ & 1.27 & $1.15-1.41$ & 1.26 & $1.16-1.36$ & 0.704 \\
\hline Hyperactivity & 3388 & 1.11 & $1.01-1.23$ & 1.21 & $1.09-1.03$ & 1.16 & $1.07-1.25$ & 0.209 \\
\hline Peer problems & 3390 & 1.19 & $1.08-1.31$ & 1.20 & $1.09-1.33$ & 1.20 & $1.11-1.29$ & 0.874 \\
\hline Anxiety & 3452 & 1.36 & $0.71-2.62$ & 1.82 & $0.98-3.36$ & 1.58 & $0.96-2.60$ & 0.474 \\
\hline Depression (at 12 years) & 3538 & 3.41 & $2.63-4.42$ & 2.71 & $2.07-3.54$ & 3.05 & $2.48-3.75$ & 0.173 \\
\hline PEs (at 12 years) & 3585 & 2.36 & $1.84-3.02$ & 4.06 & $3.20-5.15$ & 3.12 & $2.59-3.76$ & $<0.001$ \\
\hline \multicolumn{9}{|l|}{ Substance use } \\
\hline Cannabis use & 2959 & 1.23 & $1.11-1.36$ & 1.21 & $1.09-1.34$ & 1.22 & $1.13-1.32$ & 0.765 \\
\hline
\end{tabular}

OR, Odds ratio; $\mathrm{CI}$, confidence interval.

${ }^{\text {a }}$ Complete case data, bivariate model.

${ }^{\mathrm{b}} p$ values associated with significance tests comparing a model assuming psychopathology-specific effect for each exposure versus a model where the exposure effect is common/shared (i.e. constrained to be the same across psychopathologies). Small $p$ values indicate evidence of differences in fit between the two models, whereby the shared-effect model does not provide adequate fit for the data and a psychopathology-specific model provides a better fit.

sustained over time from late childhood to early adulthood $(r=0.72,95 \%$ CI $0.61-0.82)$.

\section{Common and specific risk factors}

The results for the association between each exposure and both depression and PEs at age 18 are summarized in Table 2. Most of the exposures examined within the sociodemographic, stress and adversity, emotional-behavioural and substance use risk domains were associated, to a similar degree, with an increased risk of both outcomes. This was true for low maternal education, socio-economic disadvantage and single parenthood at birth, stressful life events, peer victimization, anxiety disorder, hyperactivity, conduct problems, peer problems during childhood, and cannabis use during adolescence.

Most measures of neurodevelopment, including asphyxia at birth, early motor developmental delay, lower IQ and autistic traits, were associated with an increased risk of PEs but not depression. The likelihood ratio tests for these exposures provide support for the notion that modelling exposure effects as psychopathology specific provides a better fit to the data than modelling a common effect across depression and PEs. There was also strong evidence that PEs 
at age 12 were more strongly associated with PEs at age 18 than with depression at this age.

By contrast, depression at age 12 led to an approximately threefold increase in risk of both depression and PEs at age 18, whereas family history of depression and female sex in particular were more strongly associated with depression than PEs at age 18.

\section{MI}

Data for depression and PEs were both missing at age 12 years in $8 \%(n=555)$ of the 7030 individuals in the analysis sample, and in $37 \%(n=2595)$ at age 18 years. Approximately $50 \%(n=3524)$ had data on both depression and PE outcomes at age 12 and age 18 years. Approximately 36\% $(n=2519)$ of those with data on both depression and PE at age 12 did not have any outcome data at 18 years, whereas only $7 \%$ $(n=501)$ of those with both depression and PE data at age 18 did not have any data on either measure at age 12. Thus the main pattern for missingness was loss of follow-up. Pooled estimates from MI were similar to those using the complete-case data, and conclusions were essentially unchanged (see online Supplementary Table S1). The results were also substantively the same when examining a multivariate outcome using data on PEs and depression across both ages 12 and 18 (Supplementary Table S2).

\section{Discussion}

In this study we found evidence that most of the established risk factors for schizophrenia that we examined were associated with both PEs and depression during late adolescence within a general population sample, even after allowing for the co-morbidity between these two outcomes. Most of the exposures examined within the sociodemographic, stress and adversity, emotional-behavioural and substance use risk domains were associated, to a similar degree, with an increased risk of both outcomes. However, whereas female sex and, to a lesser extent, family history of depression showed some discrimination as potential risk factors for depression and PEs, occurring more commonly in the former, markers of abnormal neurodevelopment discriminated between these phenotypes, with stronger associations observed for PEs.

\section{Previous studies}

Studies that have examined risk factors for both PEs and depression have not been able to make direct comparisons of effects across these outcomes. For example, one cross-sectional study examined associations between sociodemographic characteristics and outcomes of psychotic symptoms and of depression while adjusting for (or conditioning on) the other outcome (Breetvelt et al. 2010). Although there was statistical evidence that ethnicity and markers of sociodemographic adversity were associated with both outcomes, and that female sex was associated with depression but not PEs, the models used do not permit formal testing of whether risk factors are shared, and to a similar extent, across both outcomes or are disorder specific. Similarly, another study (Krabbendam et al. 2004) that examined associations between risk factors and latent constructs of depression and psychosis in the general population did not test whether associations were different across these outcomes, or model these outcomes jointly to allow for the correlation between them. Given the high levels of co-morbidity between depression and PEs in general population samples (Kelleher et al. 2012a), statistical approaches that can test for differences between risk factor associations on these outcomes while allowing for their strong correlation are important.

Consistent with our findings, there is strong evidence that risk of schizophrenia is increased in individuals with neurodevelopmental abnormalities. Longitudinal studies have demonstrated that lower IQ during childhood and adolescence is associated with an increased incidence of psychotic disorders (Khandaker et al. 2011; Dickson et al. 2012), with similar findings observed for markers of motor and language development during early childhood (Welham et al. 2009a; Dickson et al. 2012). A wide variety of pregnancy and birth complications, including hypoxiarelated exposures, have been associated with risk for schizophrenia (Cannon et al. 2002b; Welham et al. 2009a), whereas genetic studies have demonstrated substantial overlap between the genetic aetiology for schizophrenia with that for autism and other neurodevelopmental disorders (Williams et al. 2010; Doherty et al. 2012). Although there is also some evidence that markers of impaired neurodevelopment (van Os et al. 1997; Zammit et al. 2004; Gale et al. 2008, 2009), autistic traits (Lundstrom et al. 2011) and birth complications (Nosarti et al. 2012) are associated with risk of depression, these results have been weaker and less consistent than those for schizophrenia (see, for example, Cannon et al. 2002a), but again no studies have directly compared associations with schizophrenia and depression within bivariate models that allow for co-morbidity of these disorders.

\section{Interpretation of findings}

Our finding, that markers of neurodevelopmental impairment are more strongly associated with PEs than with depression within such a model, supports the belief that these experiences are aetiologically related 
to pathological mechanisms underlying schizophrenia, and that their study might help us to understand more about neurodevelopmental mechanisms in the aetiology of this disorder. However, most of the exposures examined were associated with depression and PEs to the same extent. The argument that PEs are valid markers for studying the aetiology of schizophrenia, made simply on the basis that they share risk factors in common with each other, is therefore not well supported. Furthermore, although neurodevelopmental abnormalities and sex show some discrimination between PEs and depression, it is feasible that these characteristics do not discriminate PEs from other psychopathologies, such as attention deficit hyperactivity disorder, that were not examined here. Evidence supports the presence of an extended phenotype for psychosis existing across several dimensions, including an affective domain (David, 2010; Kaymaz \& van Os, 2010). Further examination of the extent to which specific characteristics distinguish PEs from other psychopathologies could lead to a greater understanding of the aetiology of psychosis.

\section{Strengths and limitations}

One of the strengths of our study is that we were able to examine and compare the effects of a wide range of risk factors measured longitudinally with respect to jointly modelled outcomes of depression and PEs. Although other studies of PEs in general population samples have described associations with IQ (Cannon et al. 2002a; Horwood et al. 2008), developmental delay (Cannon et al. 2002a), autistic traits (Bevan Jones et al. 2012) and birth complications (Zammit et al. 2009), none of these have examined whether associations with PEs are different from those for depression within multivariate models that take into account the co-morbidity or correlation of these outcomes.

One of the main limitations of our study was the substantial attrition in the cohort over time. However, the results were very similar in the imputation model that included measures of the family's socio-economic status, lifestyle and mental health, and several measures of the child's psychosocial development including data from earlier waves of depressive symptoms, PEs and other emotional and behavioural problems. Thus the assumption of missing at random conditional on these is not unrealistic.

Another limitation is that we were not able to examine all risk factors for schizophrenia, thus restricting the inferences we can make from this study. Some of the exposures examined such as anxiety disorders and family history of schizophrenia were uncommon, and the power to detect differences across psychopathologies may have been limited. Furthermore, we are not able to determine whether the associations reported here are causal as we have not explored the extent to which these associations are confounded by other variables. However, the aim of this study was not to examine causal relationships, but to explore whether characteristics relating to sociodemographic, developmental and childhood background differed between individuals with PEs and those with depression while allowing for their co-morbidity.

\section{Implications}

Longitudinal studies have demonstrated that the positive predictive value of PEs predicting even a broad phenotype of psychotic disorders is low (Poulton et al. 2000; Dhossche et al. 2002; Dominguez et al. 2011; Werbeloff et al. 2012; Zammit et al. 2013b), and this is likely to be even lower for a rarer outcome such as schizophrenia, whereas there is no evidence that PEs in population-based samples of adolescents share a genetic architecture comparable to schizophrenia (Zammit et al. 2013a). In keeping with these findings, most of the characteristics we examined, chosen for their consistent associations with schizophrenia in the literature, were associated equally strongly with depression as they were with PEs. Although PEs in the general population may have some clinical utility, given their association with depression (van Rossum et al. 2011), suicidality (Kelleher et al. 2012b) and impaired functioning (Rossler et al. 2007), in the main they seem to be a weak index of genetic and environmental risk for schizophrenia.

However, our finding that markers of neurodevelopmental impairment are more strongly associated with PEs than with depression lends some support to the belief that these phenotypes are not just alternative expressions of a common underlying mechanism, and that PEs may share some aetiological mechanisms with schizophrenia. Given their frequency and relative ease of tracking in birth cohort studies, PEs might therefore be a useful phenotype to examine mechanisms by which exposures during early brain development could impact upon risk for schizophrenia. Identification of markers that differentiate PEs from other psychopathology may enable clinicians and researchers to identify individuals with substantially higher risk of transition from PEs to clinical psychotic disorder, and where closer monitoring and earlier intervention might be warranted. More detailed measures of psychopathology over time and analytical methods appropriate for maximizing use of such data are needed to disentangle aetiological pathways to psychosis from those impacting upon co-morbid psychopathology. 


\section{Supplementary material}

For supplementary material accompanying this paper visit http://dx.doi.org/10.1017/S0033291714000026.

\section{Acknowledgments}

We are grateful to all the families who took part in this study, the midwives, and the whole ALSPAC team, including interviewers, computer and laboratory technicians, clerical workers, research scientists, volunteers, managers, receptionists and nurses.

This study was funded by Medical Research Council (MRC) grant no. G0701503. G. Lewis is a National Institute for Health Research (NIHR) Senior Investigator.

\section{Declaration of Interest}

None.

\section{References}

Amemiya T (1981). Quantitative response models: a survey. Journal of Economics Literature 19, 1483-1586.

Armando M, Nelson B, Yung AR, Ross M, Birchwood M, Girardi P, Fiori Nastro P (2010). Psychotic-like experiences and correlation with distress and depressive symptoms in a community sample of adolescents and young adults. Schizophrenia Research 119, 258-265.

Arseneault L, Cannon M, Fisher HL, Polanczyk G, Moffitt TE, Caspi A (2011). Childhood trauma and children's emerging psychotic symptoms: a genetically sensitive longitudinal cohort study. American Journal of Psychiatry 168, 65-72.

Bartels-Velthuis AA, Jenner JA, van de Willige G, van Os J, Wiersma D (2010). Prevalence and correlates of auditory vocal hallucinations in middle childhood. British Journal of Psychiatry 196, 41-46.

Bell T, Watson M, Sharp D, Lyons I, Lewis G (2005). Factors associated with being a false positive on the General Health Questionnaire. Social Psychiatry and Psychiatric Epidemiology 40, 402-407.

Bevan Jones R, Thapar A, Lewis G, Zammit S (2012). The association between early autistic traits and psychotic experiences in adolescence. Schizophrenia Research 135, 164-169.

Binbay T, Drukker M, Elbi H, Tanik FA, Ozkinay F, Onay H, Zagli N, van Os J, Alptekin K (2012). Testing the psychosis continuum: differential impact of genetic and nongenetic risk factors and comorbid psychopathology across the entire spectrum of psychosis. Schizophrenia Bulletin 38, 992-1002.

Boyd A, Golding J, Macleod J, Lawlor DA, Fraser A, Henderson J, Molloy L, Ness A, Ring S, Davey Smith G (2012). Cohort Profile: the 'children of the 90s' - the index offspring of the Avon Longitudinal Study of Parents and Children. International Journal of Epidemiology 42, 111-127.
Breetvelt EJ, Boks MP, Numans ME, Selten JP, Sommer IE, Grobbee DE, Kahn RS, Geerlings MI (2010). Schizophrenia risk factors constitute general risk factors for psychiatric symptoms in the population. Schizophrenia Research 120, 184-190.

Cannon M, Caspi A, Moffitt TE, Harrington H, Taylor A, Murray RM, Poulton R (2002a). Evidence for early-childhood, pan-developmental impairment specific to schizophreniform disorder: results from a longitudinal birth cohort. Archives of General Psychiatry 59, 449-456.

Cannon M, Jones PB, Murray RM (2002b). Obstetric complications and schizophrenia: historical and meta-analytic review. American Journal of Psychiatry 159, 1080-1092.

David AS (2010). Why we need more debate on whether psychotic symptoms lie on a continuum with normality. Psychological Medicine 40, 1935-1942.

Dhossche D, Ferdinand R, Van der Ende J, Hofstra MB, Verhulst F (2002). Diagnostic outcome of self-reported hallucinations in a community sample of adolescents. Psychological Medicine 32, 619-627.

Dickson H, Laurens KR, Cullen AE, Hodgins S (2012). Meta-analyses of cognitive and motor function in youth aged 16 years and younger who subsequently develop schizophrenia. Psychological Medicine 42, 743-755.

Doherty JL, O'Donovan MC, Owen MJ (2012). Recent genomic advances in schizophrenia. Clinical Genetics 81, 103-109.

Dominguez MD, Saka MC, Lieb R, Wittchen HU, van Os J (2010). Early expression of negative/disorganized symptoms predicting psychotic experiences and subsequent clinical psychosis: a 10-year study. American Journal of Psychiatry 167, 1075-1082.

Dominguez MD, Wichers M, Lieb R, Wittchen HU, van Os J (2011). Evidence that onset of clinical psychosis is an outcome of progressively more persistent subclinical psychotic experiences: an 8-year cohort study. Schizophrenia Bulletin 37, 84-93.

Fisher HL, Caspi A, Poulton R, Meier MH, Houts R, Harrington H, Arseneault L, Moffitt TE (2013). Specificity of childhood psychotic symptoms for predicting schizophrenia by 38 years of age: a birth cohort study. Psychological Medicine 43, 2077-2086.

Gale CR, Batty GD, Cooper C, Deary IJ (2009). Psychomotor coordination and intelligence in childhood and health in adulthood - testing the system integrity hypothesis. Psychosomatic Medicine 71, 675-681.

Gale CR, Deary IJ, Boyle SH, Barefoot J, Mortensen LH, Batty GD (2008). Cognitive ability in early adulthood and risk of 5 specific psychiatric disorders in middle age: the Vietnam experience study. Archives of General Psychiatry 65, 1410-1418.

Hanssen M, Bak M, Bijl R, Vollebergh W, van Os J (2005). The incidence and outcome of subclinical psychotic experiences in the general population. British Journal of Clinical Psychology 44, 181-191.

Harell FE (2001). Regression Modeling Strategies: With Applications to Linear Models, Logistic Regression and Survival Analysis. Springer: New York. 
Horwood J, Salvi G, Thomas K, Duffy L, Gunnell D, Hollis C, Lewis G, Menezes P, Thompson A, Wolke D, Zammit S, Harrison G (2008). IQ and non-clinical psychotic symptoms in 12-year-olds: results from the ALSPAC birth cohort. British Journal of Psychiatry 193, 185-191.

Kaymaz N, van Os J (2010). Extended psychosis phenotype-yes: single continuum-unlikely. Psychological Medicine 40, 1963-1966.

Kelleher I, Keeley H, Corcoran P, Lynch F, Fitzpatrick C, Devlin N, Molloy C, Roddy S, Clarke MC, Harley M, Arseneault L, Wasserman C, Carli V, Sarchiapone M, Hoven C, Wasserman D, Cannon M (2012a). Clinicopathological significance of psychotic experiences in non-psychotic young people: evidence from four population-based studies. British Journal of Psychiatry 201, 26-32.

Kelleher I, Lynch F, Harley M, Molloy C, Roddy S, Fitzpatrick C, Cannon M (2012b). Psychotic symptoms in adolescence index risk for suicidal behavior: findings from 2 population-based case-control clinical interview studies. Archives of General Psychiatry 69, 1277-1283.

Khandaker GM, Barnett JH, White IR, Jones PB (2011). A quantitative meta-analysis of population-based studies of premorbid intelligence and schizophrenia. Schizophrenia Research 132, 220-227.

Krabbendam L, Myin-Germeys I, De Graaf R, Vollebergh W, Nolen WA, Iedema J, van Os J (2004). Dimensions of depression, mania and psychosis in the general population. Psychological Medicine 34, 1177-1186.

Lewis G (1994). Assessing psychiatric disorder with a human interviewer or a computer. Journal of Epidemiology and Community Health 48, 207-210.

Lewis G, Pelosi AJ, Araya R, Dunn G (1992). Measuring psychiatric disorder in the community: a standardized assessment for use by lay interviewers. Psychological Medicine 22, 465-486.

Linscott RJ, van Os J (2013). An updated and conservative systematic review and meta-analysis of epidemiological evidence on psychotic experiences in children and adults: on the pathway from proneness to persistence to dimensional expression across mental disorders. Psychological Medicine 43, 1133-1149.

Little RJA, Rubin DB (2002). Statistical Analysis with Missing Data. Wiley: New York.

Lorant V, Deliege D, Eaton W, Robert A, Philippot P, Ansseau M (2003). Socioeconomic inequalities in depression: a meta-analysis. American Journal of Epidemiology 157, 98-112.

Lundstrom S, Chang Z, Kerekes N, Gumpert CH, Rastam M, Gillberg C, Lichtenstein P, Anckarsater H (2011). Autistic-like traits and their association with mental health problems in two nationwide twin cohorts of children and adults. Psychological Medicine 41, 2423-2433.

Malmberg A, Lewis G, David A, Allebeck P (1998). Premorbid adjustment and personality in people with schizophrenia. British Journal of Psychiatry 172, 308-313; discussion 314-315.

McGrath J, Saha S, Welham J, El Saadi O, MacCauley C, Chant D (2004). A systematic review of the incidence of schizophrenia: the distribution of rates and the influence of sex, urbanicity, migrant status and methodology.

BMC Medicine 2, 13.

Moore TH, Zammit S, Lingford-Hughes A, Barnes TR, Jones PB, Burke M, Lewis G (2007). Cannabis use and risk of psychotic or affective mental health outcomes: a systematic review. Lancet 370, 319-328.

Morgan C, Fisher H (2007). Environment and schizophrenia: environmental factors in schizophrenia: childhood trauma - a critical review. Schizophrenia Bulletin 33, 3-10.

Mortensen PB, Pedersen CB, Westergaard T, Wohlfahrt J, Ewald H, Mors O, Andersen PK, Melbye M (1999). Effects of family history and place and season of birth on the risk of schizophrenia. New England Journal of Medicine 340, 603-608.

Nosarti C, Reichenberg A, Murray RM, Cnattingius S, Lambe MP, Yin L, MacCabe J, Rifkin L, Hultman CM (2012). Preterm birth and psychiatric disorders in young adult life. Archives of General Psychiatry 69, 610-617.

Ntzoufras I, Dellaportas P, Forster J (2003). Bayesian variable and link determination for generalized linear models. Journal of Planning and Inference 111, 165-180.

Nuevo R, Chatterji S, Verdes E, Naidoo N, Arango C, Ayuso-Mateos JL (2012). The continuum of psychotic symptoms in the general population: a cross-national study. Schizophrenia Bulletin 38, 475-485.

Patton GC, Coffey C, Posterino M, Carlin JB, Wolfe R, Bowes G (1999). A computerised screening instrument for adolescent depression: population-based validation and application to a two-phase case-control study. Social Psychiatry and Psychiatric Epidemiology 34, 166-172.

Polanczyk G, Moffitt TE, Arseneault L, Cannon M, Ambler A, Keefe RS, Houts R, Odgers CL, Caspi A (2010). Etiological and clinical features of childhood psychotic symptoms: results from a birth cohort. Archives of General Psychiatry 67, 328-338.

Poulton R, Caspi A, Moffitt TE, Cannon M, Murray R, Harrington H (2000). Children's self-reported psychotic symptoms and adult schizophreniform disorder: a 15-year longitudinal study. Archives of General Psychiatry 57, 1053-1058.

Rossler W, Riecher-Rossler A, Angst J, Murray R, Gamma A, Eich D, van Os J, Gross VA (2007). Psychotic experiences in the general population: a twenty-year prospective community study. Schizophrenia Research 92, 1-14.

Schreier A, Wolke D, Thomas K, Horwood J, Hollis C, Gunnell D, Lewis G, Thompson A, Zammit S, Duffy L, Salvi G, Harrison G (2009). Prospective study of peer victimization in childhood and psychotic symptoms in a nonclinical population at age 12 years. Archives of General Psychiatry 66, 527-536.

Scott J, Martin G, Bor W, Sawyer M, Clark J, McGrath J (2009). The prevalence and correlates of hallucinations in Australian adolescents: results from a national survey. Schizophrenia Research 107, 179-185.

Stefanis NC, Hanssen M, Smirnis NK, Avramopoulos DA, Evdokimidis IK, Stefanis CN, Verdoux H, van Os J (2002). Evidence that three dimensions of psychosis have 
a distribution in the general population. Psychological Medicine 32, 347-358.

Thapar A, Collishaw S, Pine DS, Thapar AK (2012). Depression in adolescence. Lancet 379, 1056-1067. van Buuren S, Brand JPL, Groothuis-Oudshoorn CGM, Rubin DB (2006). Fully conditional specifications in multivariate imputation. Journal of Statistical Computation and Simulation 72, 1049-1064.

van Os J, Hanssen M, Bijl RV, Ravelli A (2000). Strauss (1969) revisited: a psychosis continuum in the general population? Schizophrenia Research 45, 11-20.

van Os J, Jones P, Lewis G, Wadsworth M, Murray R (1997). Developmental precursors of affective illness in a general population birth cohort. Archives of General Psychiatry 54, 625-631.

van Os J, Kapur S (2009). Schizophrenia. Lancet 374, 635-645. van Os J, Linscott RJ, Myin-Germeys I, Delespaul P, Krabbendam L (2009). A systematic review and meta-analysis of the psychosis continuum: evidence for a psychosis proneness-persistence-impairment model of psychotic disorder. Psychological Medicine 39, 179-195. van Rossum I, Dominguez MD, Lieb R, Wittchen HU, van Os J (2011). Affective dysregulation and reality distortion: a 10-year prospective study of their association and clinical relevance. Schizophrenia Bulletin 37, 561-571.

Varghese D, Scott J, Welham J, Bor W, Najman J, O'Callaghan M, Williams G, McGrath J (2011).

Psychotic-like experiences in major depression and anxiety disorders: a population-based survey in young adults. Schizophrenia Bulletin 37, 389-393.

Welham J, Isohanni M, Jones P, McGrath J (2009a). The antecedents of schizophrenia: a review of birth cohort studies. Schizophrenia Bulletin 35, 603-623.

Welham J, Scott J, Williams G, Najman J, Bor W, O'Callaghan M, McGrath J (2009b). Emotional and behavioural antecedents of young adults who screen positive for non-affective psychosis: a 21-year birth cohort study. Psychological Medicine 39, 625-634.

Werbeloff N, Drukker M, Dohrenwend BP, Levav I, Yoffe R, van Os J, Davidson M, Weiser M (2012). Self-reported attenuated psychotic symptoms as forerunners of severe mental disorders later in life. Archives of General Psychiatry 69, 467-475.

Wicks S, Hjern A, Gunnell D, Lewis G, Dalman C (2005). Social adversity in childhood and the risk of developing psychosis: a national cohort study. American Journal of Psychiatry 162, 1652-1657.

Wiles NJ, Zammit S, Bebbington P, Singleton N, Meltzer H, Lewis G (2006). Self-reported psychotic symptoms in the general population: results from the longitudinal study of the British National Psychiatric Morbidity Survey. British Journal of Psychiatry 188, 519-526.

Williams NM, Zaharieva I, Martin A, Langley K, Mantripragada K, Fossdal R, Stefansson H, Stefansson K, Magnusson P, Gudmundsson OO, Gustafsson O, Holmans P, Owen MJ, O'Donovan M, Thapar A (2010). Rare chromosomal deletions and duplications in attention-deficit hyperactivity disorder: a genome-wide analysis. Lancet 376, 1401-1408.

Zammit S, Allebeck P, David AS, Dalman C, Hemmingson T, Lundberg I, Lewis G (2004). A longitudinal study of premorbid IQ score and risk of developing schizophrenia, bipolar disorder, severe depression, and other nonaffective psychoses. Archives of General Psychiatry 61, 354-360.

Zammit S, Hamshere M, Dwyer S, Georgiva L, Timpson N, Moskvina V, Richards A, Evans DM, Lewis G, Jones PB, Owen MJ, O'Donovan MC (2013a). A population-based study of genetic variation and psychotic experiences in adolescents. Schizophrenia Bulletin. Published online: 30 October 2013. doi: 10.1093/schbul/sbt146.

Zammit S, Horwood J, Thompson A, Thomas K, Menezes P, Gunnell D, Hollis C, Wolke D, Lewis G, Harrison G (2008). Investigating if psychosis-like symptoms (PLIKS) are associated with family history of schizophrenia or paternal age in the ALSPAC birth cohort. Schizophrenia Research 104, 279-286.

Zammit S, Kounali D, Cannon M, David AS, Gunnell D, Heron J, Jones PB, Lewis S, Sullivan S, Wolke D, Lewis G (2013b). Psychotic experiences and psychotic disorders at age 18 in relation to psychotic experiences at age 12 in a longitudinal population-based cohort study. American Journal of Psychiatry 170, 742-750.

Zammit S, Odd D, Horwood J, Thompson A, Thomas K, Menezes P, Gunnell D, Hollis C, Wolke D, Lewis G, Harrison G (2009). Investigating whether adverse prenatal and perinatal events are associated with non-clinical psychotic symptoms at age 12 years in the ALSPAC birth cohort. Psychological Medicine 39, 1457-1467. 